\title{
Glaucoma aǵudo bilateral em paciente jovem secundário ao uso de topiramato: relato de caso
}

\author{
Bilateral acute angle closure glaucoma in a young patient recei ving oral topiramate: \\ case report
}

\author{
Fausto Stangler ${ }^{1}$ \\ Roberta Fernandez Prietsch ${ }^{2}$ \\ João Borges Fortes Filho ${ }^{3}$
}

Trabalho realizado no curso de especialização em oftalmologia do Hospital Banco de Olhos de Porto Alegre - Porto Alegre (RS) - Brasil.

${ }^{1}$ Médico Oftalmologista, Ex-residente do Curso de Especialização em Oftalmologia no Hospital Banco de Olhos de Porto Alegre - Porto Alegre (RS) - Brasil.

${ }^{2}$ Residente do Curso de Especialização em Oftalmologia do Hospital Banco de Olhos de Porto Alegre - Porto Alegre (RS) - Brasil

${ }^{3}$ Professor de Oftalmologia da Faculdade de Medicina da Universidade Federal do Rio Grande do Sul - UFRGS

- Porto Alegre (RS) - Brasil. Doutorando em Oftalmologia pela Universidade Federal de São Paulo - UNIFESP - São Paulo (SP) - Brasil. Coordenador do Curso de Especialização em Oftalmologia do Hospital Banco de Olhos de Porto Alegre - Porto Alegre (RS) - Brasil.

Endereco para correspondência: João Borges Fortes Filho. Hospital Banco de Olhos de Porto Alegre. Rua Eng. Walter Boehl, 285 - Porto Alegre (RS) CEP 91360-090

E-mail: jbfortes@cursohbo.com.br

Recebido para publicação em 26.06.2005

Última versão recebida em 22.03.2006

Aprovação em 06.04.2006

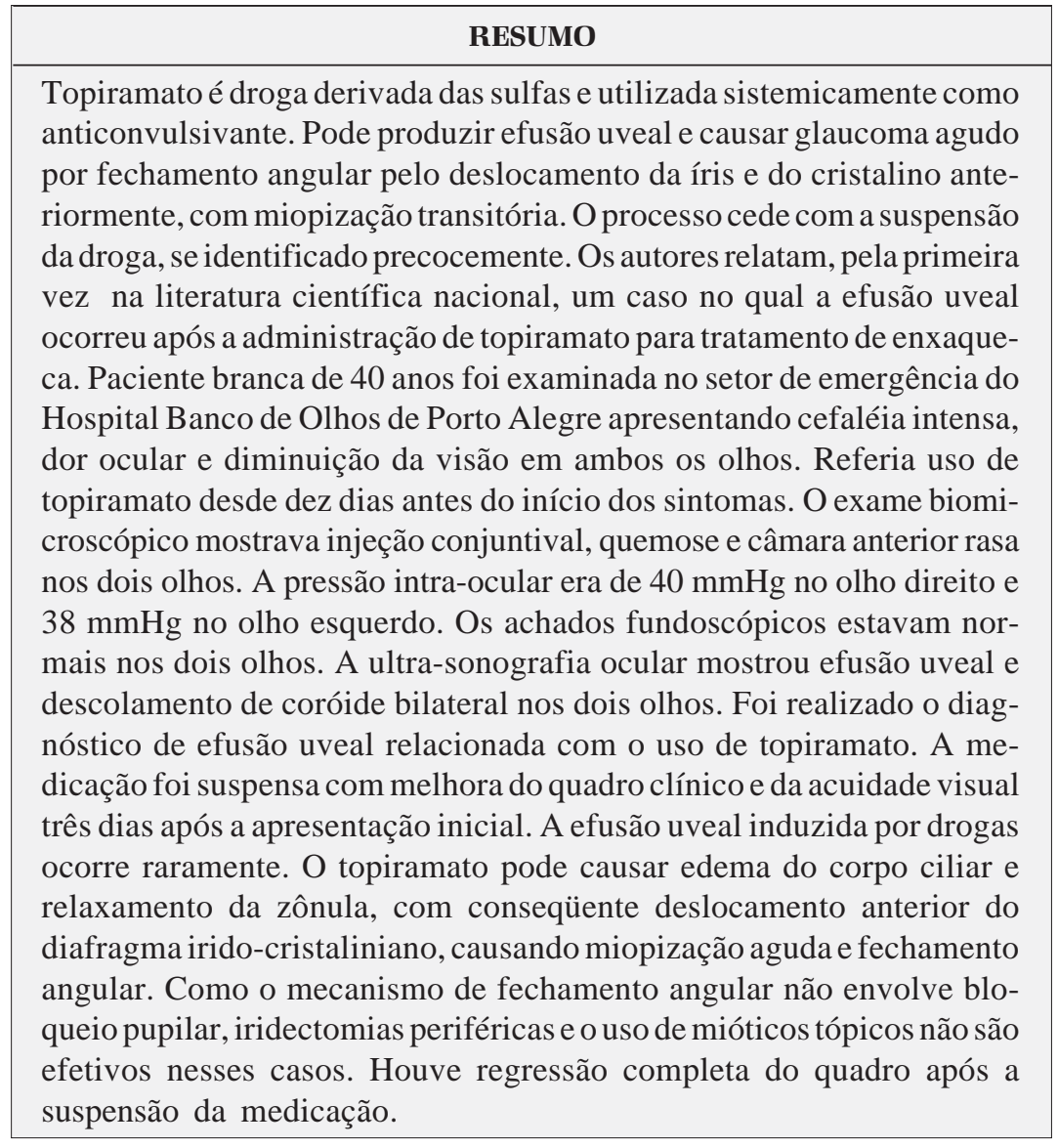

Descritores: Glaucoma de ângulo fechado/induzido quimicamente; Frutose/efeitos adversos; Anticonvulsivos/efeitos adversos; Pressão intra-ocular; Doenças da úvea/ induzido quimicamente

\section{INTRODUÇÃOO}

Existem várias drogas de uso sistêmico capazes de causar glaucoma agudo. Este artigo descreve pela primeira vez na literatura científica nacional a ocorrência de glaucoma agudo por fechamento angular nos dois olhos de uma paciente jovem como efeito adverso ao uso do topiramato, uma droga anticonvulsivante derivada das sulfas e de amplo uso como anticonvulsivante nos dias de hoje. São discutidos a fisiopatologia e o manejo deste tipo de glaucoma que é totalmente reversível se identificado precocemente.

O glaucoma de ângulo fechado pode ser classificado em primário ou secundário e agudo ou crônico. São numerosos os processos fisiopatológi- 
cos envolvidos em cada uma dessas entidades ${ }^{(1)}$. O glaucoma secundário de ângulo fechado pode se apresentar em uma ampla variedade de situações clínicas, mas identificar o processo fisiopatológico causador do ataque agudo de glaucoma nem sempre é simples. Ele pode ser causado, como relatado a seguir, por medicamentos de uso comum na prática médica.

O topiramato é uma droga anticonvulsivante que tem como efeito raro uma síndrome caracterizada por miopização e glaucoma agudo bilateral. A etiologia do processo é um edema do corpo ciliar com consequiente deslocamento anterior do diafragma irido-cristaliniano, câmara anterior rasa e fechamento do seio camerular sem bloqueio pupilar. A suspensão da droga se faz necessária para a reversão do quadro.

\section{RELATO DO CASO}

Paciente de 40 anos, sexo feminino, procurou a emergência do Hospital Banco de Olhos de Porto Alegre por dor ocular em ambos os olhos há quase 36 horas. Mencionava a visão de halos coloridos ao redor das luzes e diminuição da acuidade visual, além de cefaléia intensa. Os sintomas iniciaram primeiramente no olho direito, mas logo após, manifestaram-se também no olho esquerdo. A paciente utilizou, no dia anterior, antiinflamatórios não hormonais via oral para a dor. Ela havia ido a uma consulta oftalmológica seis meses antes quando foram receitadas lentes de $+0,50$ dioptrias esféricas para ambos os olhos.

Em relação a antecedentes pessoais sistêmicos, relatou crises de enxaqueca e fazia uso de Topamax $^{\circledR}$ (topimarato) para a profilaxia das crises. Negava outras doenças ou uso de outras medicações.

Ao exame oftalmológico, apresentava acuidade visual sem correção de contar dedos a três metros no olho direito e 20/400 no olho esquerdo, mas mantinha boa acuidade visual para perto (J2) com os dois olhos. A retinoscopia do olho direito mostrava -4,50 dioptrias esféricas. Não foi possível realizar a retinoscopia do olho esquerdo.

Havia importante quemose conjuntival bilateral embora esta não se evidencie tão claramente na imagem da figura 1. À biomicroscopia, notavam-se pupilas mióticas, mas fotorreagentes. A câmara anterior era rasa, a córnea clara e o humor aquoso sem alterações em ambos os olhos (Figuras 2 e 3). A pressão intra-ocular, por ocasião da primeira consulta, foi de $40 \mathrm{mmHg}$ no olho direito e $38 \mathrm{mmHg}$ no olho esquerdo.

Com base na história clínica e no exame físico, foi estabelecido o diagnóstico clínico de glaucoma agudo bilateral. A paciente foi tratada inicialmente com os colírios Cosopt ${ }^{\circledR}$, pilocarpina $2 \%$ e Predfort ${ }^{\circledR}$ além de acetazolamida VO e manitol EV. Após uma hora e meia do início da medicação a pressão intraocular caiu para $24 \mathrm{mmHg}$ em ambos os olhos. Por se tratar de apresentação bilateral em paciente jovem, foi cogitada a hipóte-

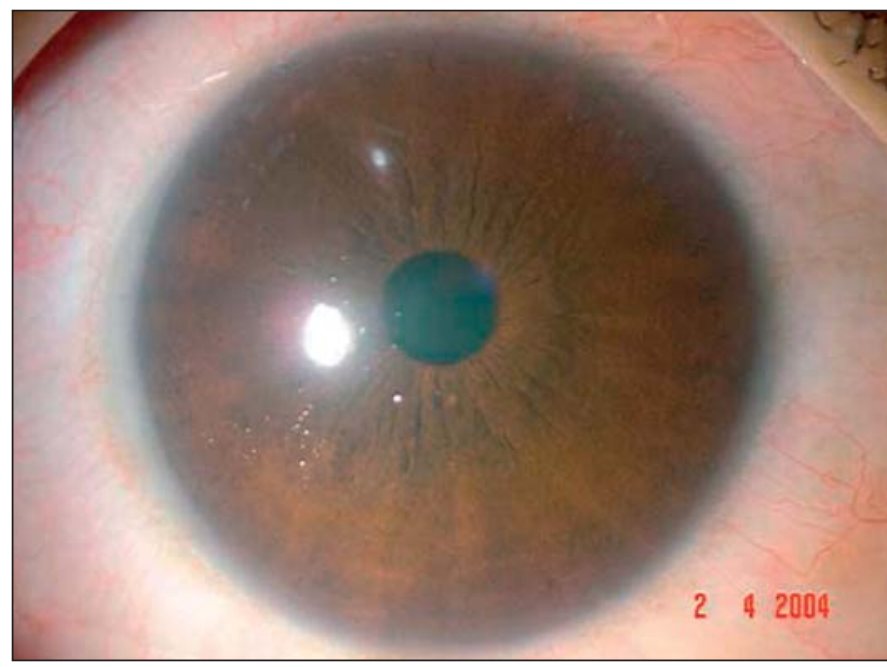

Figura 1 - Discreta quemose conjuntival, pupila em miose e edema de córnea por ocasião da primeira consulta no dia 2/4/2004

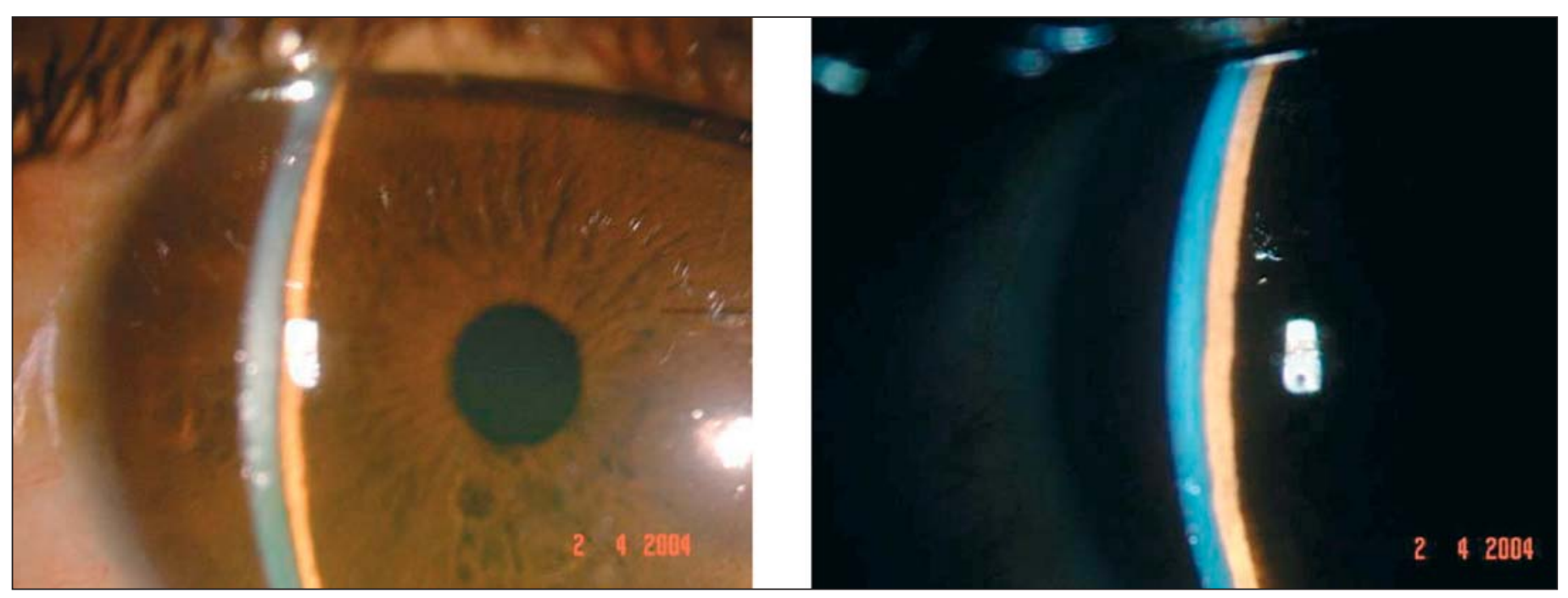

Figuras 2 e 3 - Câmara anterior muito rasa e edema de córnea, por ocasião da primeira consulta no dia 2/4/2004 
se de glaucoma agudo secundário ao uso de topiramato, o que justificou sua suspensão a partir daquele momento.

No mesmo dia, após a resposta favorável à medicação inicialmente administrada, foi realizada ecografia ocular, que mostrou descolamento periférico de coróide e efusão uveal supraciliar em ambos os olhos (Figuras 4 e 5).

Três dias após o primeiro atendimento na emergência, a paciente encontrava-se sem dor ocular, com acuidade visual de 20/25 sem correção em ambos os olhos. À biomicroscopia, a câmara anterior era profunda, a córnea estava clara e não havia sinais de quemose conjuntival. O fundo de olho não apresentava alterações. Somente então, foi possível realizar a gonioscopia, que mostrou ângulo da câmara anterior aberto em ambos os olhos (Figuras 6 e 7). A pressão intra-ocular agora era de $4 \mathrm{mmHg}$ em ambos os olhos. A medicação para a crise foi suspensa no terceiro dia e a paciente foi avaliada no sétimo e no décimo quinto dias após a apresentação inicial. Em ambas as ocasiões não apresentava nenhum sinal de elevação da pressão intra-ocular, nem de ângulo estreito ou de alterações do disco óptico, tendo sido dado por encerrado seu tratamento.

\section{DISCUSS $\tilde{A} O$}

O topiramato é uma nova droga anticonvulsivante que tem sido prescrita com muita freqüência em todo o mundo. Além do uso primário, tem demonstrado eficácia no tratamento de transtornos bipolares e na profilaxia da enxaqueca tanto em adultos como em crianças $^{(2)}$. Derivada das sulfas, esta droga bloqueia os neurotransmissores por inibição dos canais de cálcio potencializando a GABA-A (inibidor da neurotransmissão) e bloqueando a ativação dos receptores excitatórios do glutamato $^{(3)}$.

Uma síndrome caracterizada por miopia transitória e glaucoma agudo tem sido atribuída ao uso desta medicação. Os sinais
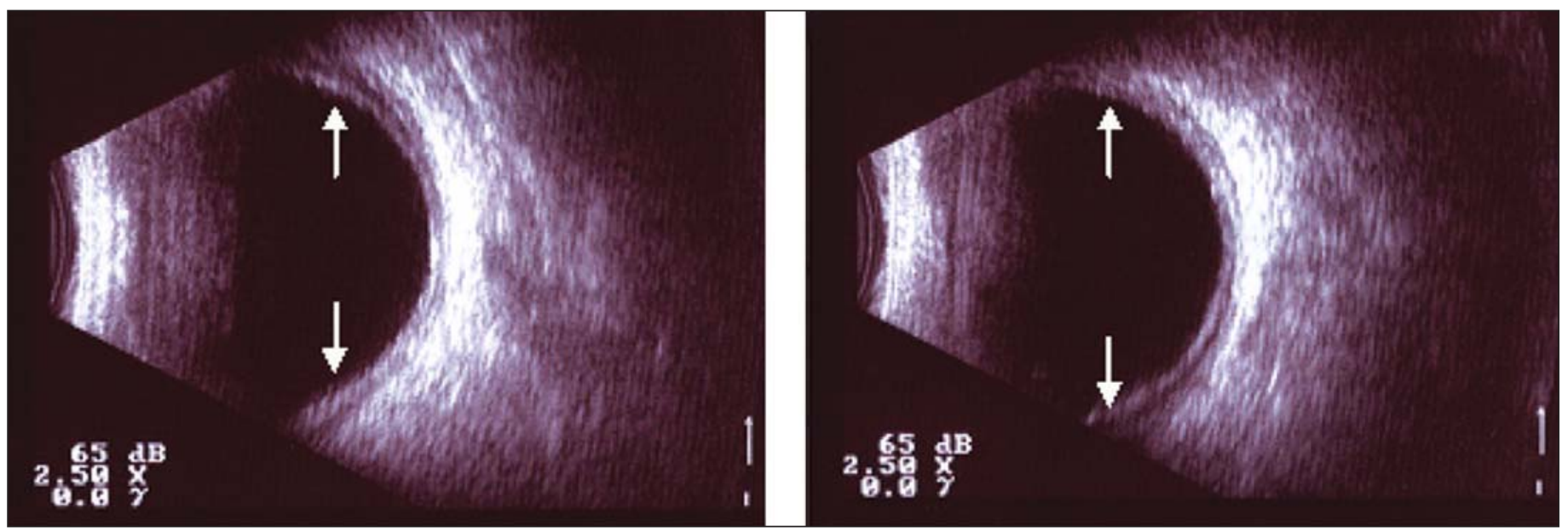

Figuras 4 e 5 - Ecografia ocular mostrando descolamento periférico de coróide e efusão uveal supraciliar em ambos os olhos

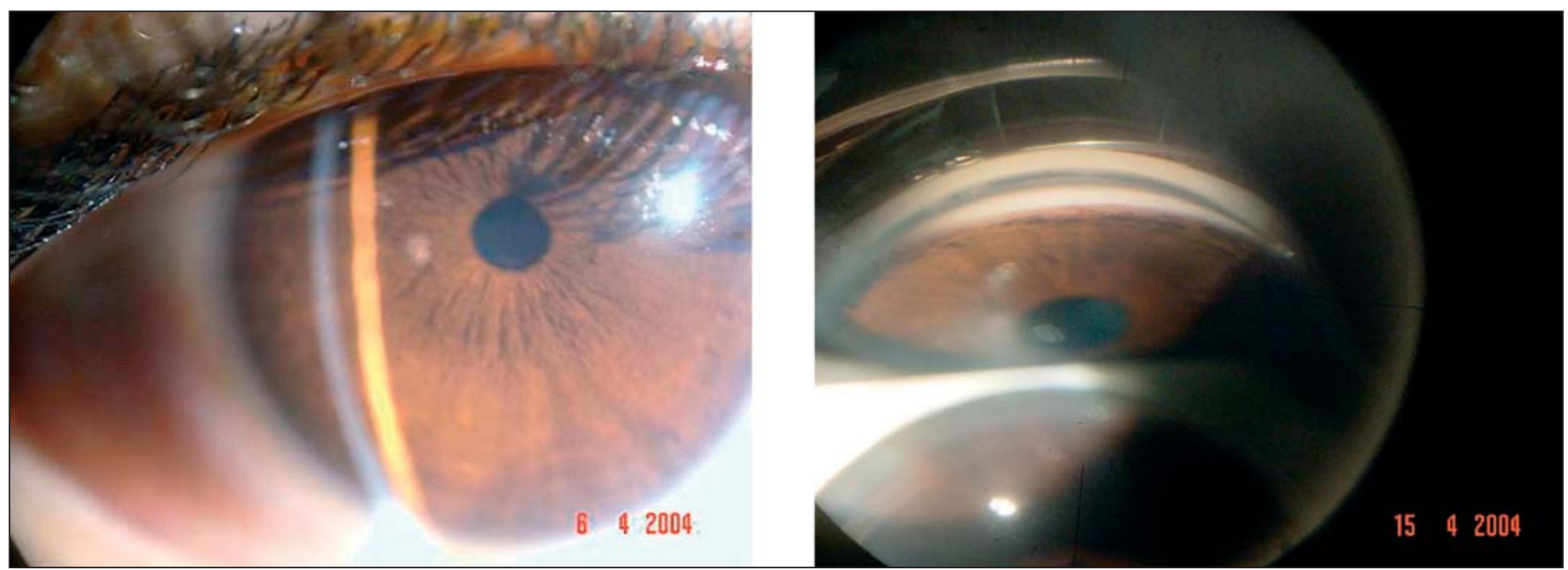

Figuras 6 e 7 - Câmara anterior mais profunda no dia 6/4/2004, após a suspensão do topiramato. Goniofotografia obtida no dia 15/4/2004 mostrando ângulo aberto e pigmentação na malha trabecular após a resolução do quadro clínico. 
e sintomas ocorrem tipicamente durante o primeiro mês de tratamento, e incluem decréscimo na acuidade visual, dor ocular, estreitamento da câmara anterior, hiperemia e aumento da pressão intra-ocular. Pode ocorrer também diplopia e nistagmo ${ }^{(3)}$.

Ao contrário do glaucoma agudo primário, mais prevalente na sexta e sétima décadas ${ }^{(1)}$, o glaucoma causado pelo uso do topiramato em geral é bilateral e já foi descrito em pacientes de meia-idade e até mesmo em crianças ${ }^{(2,4-5)}$.

Embora existam controvérsias a respeito do mecanismo causador do glaucoma agudo e da miopia, acredita-se que a efusão uveal favoreça o relaxamento da zônula, causando deslocamento anterior do diafragma irido-cristaliniano. Como consequiência, ocorreria miopização e uma redução da profundidade da câmara anterior. O descolamento da coróide está freqüentemente associado ao quadro clínico ${ }^{(5-6)}$. Alguns autores sugerem que a miopia aguda pode também estar relacionada com a fraca inibição da anidrase carbônica causada pelo topiramato, com o edema do cristalino e o espasmo acomodativo tendo como conseqüência a miopização ${ }^{(3,7)}$.

A efusão uveal induzida por drogas é extremamente rara, embora tenha sido relatada também com o uso de derivados das sulfas, como a acetazolamida, hidroclortiazida, sulfametoxazol e os inibidores seletivos da recaptação de serotonina ${ }^{(2,4,6,8-9)}$.

O quadro clínico é reversível se diagnosticado precocemente. A suspensão imediata da droga é imprescindível, sendo necessário uma interação com o clínico para que se possa buscar uma droga alternativa para o tratamento da patologia de base.

Como o glaucoma agudo, nesse caso, é causado por efusão uveal sem bloqueio pupilar, as iridotomias periféricas e os mióticos são, portanto, ineficazes para o tratamento podendo, inclusive, agravar o quadro clínico, por deslocar a íris e o cristalino ainda mais anteriormente $\mathrm{e}^{(2,5-6)}$.

É conhecido o fato de que a elevação aguda da pressão intra-ocular por períodos prolongados, qualquer que seja sua etiologia, leva a um dano severo e irreversível do nervo óptico. É necessário que o oftalmologista procure não somente tratar a crise de glaucoma, mas também e sempre que possível, busque as suas causas. Somente assim será possível estabelecer a estratégia mais adequada para o manejo de cada paciente, evitando iatrogenias adicionais e atuando eficazmente para a sua mais pronta recuperação conforme foi realizado com esta paciente que recuperou a profundidade da câmara anterior e teve normalizada sua pressão intra-ocular nos dois olhos após a suspensão da medicação (topiramato).

\section{ABSTRACT}

This paper reports a clinical case of uveal effusion in both eyes causing bilateral acute angle closure glaucoma in a young patient after oral administration of topiramate, a new anticonvulsivant medication. Rarely, some drugs have produced uveal effusions, forward shift of the iris-lens diaphragm, transient myopia and secondary angle closure glaucoma. A 40-year old white woman was seen at the emergency department of the "Hospital Banco de Olhos de Porto Alegre (RS)" Brazil, with severe headaches and blurry vision in both eyes. Her medications included topiramate, started 10 days before. Slit lamp examination revealed conjunctival injection, chemosis and shallow anterior chambers. Intraocular pressure measured 40 and $38 \mathrm{mmHg}$. Fundoscopic examination findings were normal. Ultrasound scan was performed and demonstrated separation between the choroidal layer and the sclera. A diagnosis of bilateral uveal effusion associated with the use of oral topiramate was made. Topiramate was then discontinued. The patient reported symptomatic improvement by the third day after initial examination. Symptoms were resolved and visual acuity returned to normal. Topiramate may cause ciliary body edema and relaxation of zonules, which induces a forward shift of the lens-iris diaphragm with acute myopia and angle closure. As the mechanism of angle closure does not involve pupillary block, peripheral iridectomy and topical miotics are not useful in the treatment of this type of secondary angle-closure glaucoma. Drug-induced uveal effusions occur rarely. The patient improved after topiramate discontinuation.

Keywords: Glaucoma, angle closure/chemically induced; Fructose/adverse effects; Anticonvulsants/adverse effects; Intraocular pressure; Uveal diseases/chemically induced

\section{REFERÊNCIAS}

1. Malta RFS. Glaucoma de ângulo estreito. In: Susanna Junior RS. Glaucoma. Manual do Conselho Brasileiro de Oftalmologia. São Paulo: Cultura Médica; 1999. p.123-44.

2. Medeiros FA, Zhang XY, Bernd AS, Weinreb RN. Angle-closure glaucoma associated with ciliary body detachment in patients using topiramate. Arch Ophthalmol. 2003;121(2):282-5.

3. Gubbay SS. The occurrence of drug-induced myopia as a transient side effect of topiramate. Epilepsia. 1998;39(4):451.

4. Sankar PS, Pasquale LR, Grosskreutz CL. Uveal effusion and secondary angle-closure glaucoma associated with topiramate use. Arch Ophthalmol. 2001;119(8):1210-1. Comment in: Arch Ophthalmol. 2002;120(8):1108.

5. Rhee DJ, Goldberg MJ, Parrish RK. Bilateral angle-closure glaucoma and ciliary body swelling from topiramate. Arch Ophthalmol. 2001;119(11):1721-3. Comment in: Arch Ophthalmol. 2002;120(12):1775.

6. Banta JT, Hoffman K, Budenz DL, Ceballos E, Greenfield DS. Presumed topiramate-induced bilateral acute angle-closure glaucoma. Am J Ophthalmol. 2001;132(1):112-4.

7. Postel EA, Assalian A, Epstein DL. Drug-induced transient myopia and angleclosure glaucoma associated with supraciliary choroidal effusion. Am J Ophthalmol. 1996;122(1):110-2.

8. Fan JT, Johnson DH, Burk RR. Transient myopia, angle-closure glaucoma, and choroidal detachment after oral acetazolamide. Am J Ophthalmol. 1993; 115(6):813-4

9. Lin J, Fosnot J, Edmond J. Bilateral angle closure glaucoma in a child receiving oral topiramate. J AAPOS. 2003;7(1):66-8. Comment in: J AAPOS. 2003;7(4):303 